\title{
Amygdala Central Nucleus Interacts with Dorsolateral Striatum to Regulate the Acquisition of Habits
}

\author{
Nura W. Lingawi and Bernard W. Balleine \\ Brain and Mind Research Institute, University of Sydney, Camperdown, New South Wales 2050, Australia
}

The role of the amygdala central nucleus (CeN) in habit learning was assessed in two experiments. First, we examined the effects of bilateral lesions of the anterior $\mathrm{CeN}$ on an overtraining-induced lever press habit evaluated using an outcome devaluation protocol. Overtraining generated habitual performance and rendered sham lesioned rats insensitive to outcome devaluation, an effect that was also found in rats given control lesions of the posterior CeN. In contrast, rats with lesions of the anterior CeN did not show normal habit acquisition and their performance remained goal-directed and sensitive to outcome devaluation. Nevertheless, lesions of either the posterior or the anterior $\mathrm{CeN}$ abolished the general excitatory influence of a Pavlovian conditioned stimulus on instrumental performance. Second, we assessed the functional interaction between the CeN and dorsolateral striatum (DLS), a region previously implicated in the acquisition of habits, using asymmetrical lesions to disconnect these structures. Rats were given a unilateral lesion of anterior $\mathrm{CeN}$ and a unilateral lesion of the DLS, made either ipsilateral (control) or contralateral (disconnection) to the CeN lesion, and given overtraining followed by outcome devaluation. Although the ipsilateral lesioned rats were insensitive to devaluation, the contralateral CeN-DLS lesion impaired habit acquisition, rendering performance sensitive to the devaluation treatment. These results are the first to implicate the CeN and its connection with a circuit involving DLS in habit learning. They imply that, in instrumental conditioning, regions of amygdala parse the instrumental outcome into the reward and reinforcement signals mediating goal-directed and habitual actions, respectively.

\section{Introduction}

Considerable evidence suggests that instrumental conditioning is governed by two learning processes (Dickinson et al., 1995; Balleine and Dickinson, 1998). During initial acquisition, instrumental performance depends on encoding the relationship between an action and its consequences or outcome as well as the current value of the outcome (Adams and Dickinson, 1981; Balleine and Dickinson, 1998). After a period of overtraining, however, actions can become inflexible or habitual, are no longer sensitive to changes in outcome value, and appear to come under the control of antecedent stimuli rather than their consequences (Adams, 1982; Dickinson et al., 1995). The acquisition of stimulus control appears, however, to differ from that associated with goal-directed learning: rather than involving error-correction learning, it appears to depend on both stimulus-response (S-R) contiguity and a reinforcement signal that acts as a catalyst to strengthen the S-R association as training progresses (Balleine

\footnotetext{
Received Sept. 21, 2011; revised Nov. 6, 2011; accepted Nov. 8, 2011.

Author contributions: N.W.L. and B.W.B. designed research; N.W.L. performed research; N.W.L. and B.W.B. analyzed data; N.W.L. and B.W.B. wrote the paper.

The work was supported by grants from the National Institute of Mental Health (MH56446), the National Health and Medical Research Council (633267), and a Laureate Fellowship from the Australian Research Council. We thank Dr Vincent Laurent for his comments on the manuscript.

Correspondence should be addressed to Bernard W. Balleine, Behavioural Neuroscience Laboratory, Brain and Mind Research Institute, University of Sydney, Level 6, 100 Mallet Street, Camperdown, New South Wales 2050, Australia. E-mail: Bernard.Balleine@sydney.edu.au.

DOI:10.1523/JNEUROSCI.4806-11.2012

Copyright $\odot 2012$ the authors $\quad 0270-6474 / 12 / 321073-09 \$ 15.00 / 0$
}

and Dickinson, 1998; Dayan and Balleine, 2002; Balleine and Killcross, 2006).

The dorsal striatum plays a pivotal role in both these learning processes: pharmacological manipulations that block plasticity in dorsomedial striatum (DMS) abolish the acquisition and performance of goal-directed actions (Yin et al., 2005a; Shiflett et al., 2010) whereas lesion or inactivation of the dorsolateral striatum (DLS) (Yin et al., 2004, 2006) or of its dopamine afferents from the substantia nigra pars compacta (SNc) (Faure et al., 2005) have been found to block the acquisition and performance of habits. In addition to the DMS, the amygdala, specifically the basolateral nucleus (BLA), plays a critical role in the acquisition of goaldirected actions: lesion, inactivation, and pharmacological manipulations of the BLA have been found to block sensitivity of instrumental performance to changes in outcome value (Balleine et al., 2003; Ostlund and Balleine, 2008; Wassum et al., 2009). The role of the amygdala in habit learning, however, remains to be assessed.

There are several reasons to predict that the amygdala, specifically the central nucleus $(\mathrm{CeN})$, plays a role in habits. First, evidence suggests that the nigrostriatal projection to the DLS provides the reinforcement signal for S-R learning (Reynolds et al., 2001; Faure et al., 2005) and the CeN, particularly its anterior efferents (Gonzales and Chesselet, 1990), appears to be a key modulator of SNc function (Rouillard and Freeman, 1995; Fudge and Emiliano, 2003). Other studies have implicated $\mathrm{CeN}$ in the stimulus control of responses, such as orienting (El-Amamy and Holland, 2006), and in the general excitatory effect of Pavlovian stimuli on instrumental performance (Holland and Gallagher, 
2003; Corbit and Balleine, 2005). The amygdala may, therefore, play a general role in action control, parsing instrumental outcomes into the reward (BLA) and reinforcement $(\mathrm{CeN})$ signals that mediate goal-directed and habit learning, respectively. The current experiments sought to assess this hypothesis by comparing the effects of (1) lesions of anterior $\mathrm{CeN}$, a region with high connectivity to the $\mathrm{SNc}$, with both sham and posterior $\mathrm{CeN}$ lesioned controls; and (2) disconnection of the anterior $\mathrm{CeN}$ from the DLS using asymmetrical lesions on habits, assessed by the sensitivity of performance to taste aversion-induced outcome devaluation (Adams, 1982).

\section{Materials and Methods}

\section{Overview of experiments}

Two separate experiments were conducted in this study. Experiment 1 assessed the effects of ibotenic acid-induced lesions of the anterior CeN on overtraining-induced habits against the performance of two control groups: a sham group and a group given lesions of the posterior $\mathrm{CeN}$. To assess the functional influence of the lesions in the posterior $\mathrm{CeN}$ group, we also incorporated a test of general Pavlovian-instrumental transfer into this experiment. As a consequence, this experiment was conducted in four phases: instrumental training, devaluation by taste aversion learning, an extinction test of outcome devaluation, and the assessment of general transfer.

Experiment 2 assessed the effect of disconnection of the CeN from the dorsolateral striatum in habit learning. Two groups were used: a control group given lesions of both the anterior $\mathrm{CeN}$ and dorsolateral striatum in the same hemisphere and an experimental group given lesions in contralateral hemispheres. The experiment was conducted in three phases: instrumental training, devaluation by taste aversion learning, and devaluation extinction test.

\section{Subjects}

Male outbred Long-Evans rats $(n=165)$ obtained from the Laboratory Animal Services (University of Sydney, Australia) were used for Experiments 1 and 2. All rats weighed between 290 and $340 \mathrm{~g}$ and were housed in pairs in a humidity- and temperature-controlled colony room. Rats were maintained on a $12 \mathrm{~h} \mathrm{light/dark} \mathrm{cycle} \mathrm{(7:00} \mathrm{A.M} \mathrm{to} \mathrm{7:00} \mathrm{P.M.)} \mathrm{and}$ were handled daily 1 week before the commencement of the experiment. Throughout the experiments, rats were maintained at $\sim 85 \%$ of their free-feeding body weight by restricting their food intake. The University of Sydney Animal Ethics Committee approved all experimental procedures.

\section{Apparatus}

All behavioral procedures were performed in 16 identical Med Associates operant chambers enclosed in sound- and light-attenuating shells. Each chamber was equipped with a pump that was fitted with a syringe that delivered $20 \%$ sucrose solution $(0.1 \mathrm{ml})$ and a pellet dispenser that delivered grain pellets ( $45 \mathrm{mg}$; Bio Serve) into a recessed food magazine. An infrared photobeam that crossed the magazine provided for the detection of magazine head entries. Each chamber contained two retractable levers to the right and left of the magazine and a $3 \mathrm{~W} 24 \mathrm{~V}$ houselight mounted on the top of the wall opposite the magazine provided illumination. Chambers were also equipped with a sonalert that delivered a $3 \mathrm{kHz}$ tone and a white-noise generator. All auditory stimuli were adjusted to $80 \mathrm{~dB}$ in the presence of $60 \mathrm{~dB}$ background noise provided by a ventilation fan. Two microcomputers running on the Med-PC program (Med Associates) controlled experimental events and recorded lever presses and magazine entries.

\section{Surgery}

Rats were allowed ad libitum access to food and water before and for $5 \mathrm{~d}$ following surgery. At the time of surgery, rats were anesthetized with isoflurane (5\% for induction and $2-3 \%$ for maintenance) and treated with a subcutaneous injection of $0.1 \mathrm{ml}$ of Rymadil and an intraperitoneal injection of $0.4 \mathrm{ml}(300 \mathrm{mg} / \mathrm{kg})$ solution of procaine penicillin. Rats were placed in a stereotaxic frame (Stoelting). An incision was made to expose the scalp and the incisor bar was adjusted to align bregma and lambda on the same horizontal plane. For all rats, holes were drilled into the skull above the appropriate targeted structures according to the following coroordinates: the anterior $\mathrm{CeN}(\mathrm{AP},-1.8 ; \mathrm{ML}, \pm 4.0 ; \mathrm{DV},-7.7$ relative to bregma), the posterior $\mathrm{CeN}(\mathrm{AP},-2.3 ; \mathrm{ML}, \pm 4.0 ; \mathrm{DV},-7.6$ $\mathrm{mm}$, relative to bregma), and the DLS (AP, 0.7; $\mathrm{ML}, \pm 3.6$ relative to bregma; DV, -5.0 relative to skull surface). Each infusion was made using a $1 \mu \mathrm{l}$ Hamilton syringe. Injections and of ibotenic acid $(0.06 \mathrm{M})$ over $4 \mathrm{~min}$ were used to make anterior $(0.15 \mu \mathrm{l})$ and posterior $(0.2 \mu \mathrm{l})$ lesions. DLS lesions were made by infusing $0.3 \mu$ l of NMDA $(0.12 \mathrm{M})$ over $5 \mathrm{~min}$. The needle was left in place for $3 \mathrm{~min}$ after each infusion to allow for the diffusion of the drug.

Rats receiving sham lesions had holes drilled in the skull but the needle was not lowered and no drug was infused. Rats in Experiment 1 received bilateral anterior $\mathrm{CeN}$, posterior $\mathrm{CeN}$, or sham lesions. Rats in Experiment 2 received both a unilateral anterior CeN and a unilateral DLS lesion. For half of the rats in Experiment 2, the DLS was lesioned in the hemisphere ipsilateral to the CeN lesion; for the other half, the DLS lesion was contralateral to the $\mathrm{CeN}$ lesion.

We conducted Experiment 1 in two parts. Experiment 1A compared posterior CeN-lesioned rats ( $\mathrm{pCeN} ; n=24)$ against sham controls $(n=$ 23). Experiment $1 \mathrm{~B}$ compared $\mathrm{pCeN}(n=22)$, anterior CeN-lesioned rats $(\mathrm{aCeN} ; n=22)$, and sham controls $(n=22)$. Experiment 2 compared two groups: a contralateral-lesioned group (Contra; $n=26$ ) and an ipsilateral-lesioned control (Ipsi; $n=26)$.

\section{Histological analysis}

After behavioral testing, all rats were deeply anesthetized by $0.3 \mathrm{ml}$ intraperitoneal injection of pentobarbitone sodium $(325 \mathrm{mg} / \mathrm{ml})$ and perfused transcardinally with $4 \%$ formaldehyde solution. Brains were stored in $4 \%$ formaldehyde for $24 \mathrm{~h}$ and were then transferred to a $20 \%$ sucrose solution in PBS ( $\mathrm{pH}, 7.2$ ). Alternating $40 \mu \mathrm{m}$ coronal sections were then taken and placed in separate wells (10-15 sections per well) containing PBS.

Sections containing amygdala and striatal structures were initially placed in a solution of $0.1 \%$ Triton X-100 and PBS for 10 min. Sections were washed twice for $5 \mathrm{~min}$ in PBS and then incubated in NeuroTrace Fluorescent Nissl (1:50; Invitrogen) for $60 \mathrm{~min}$. Sections were then placed in $0.1 \%$ Triton X-100 and PBS for 10 min, washed in PBS twice for 5 min, and mounted with Fluromount mounting medium (Southern Biotech).

A second series of amygdala sections were stained with glial fibrillary acidic protein (GFAP), a marker for astrocytes. Sections were placed in PBS with $0.1 \%$ Triton X-100 for $10 \mathrm{~min}$, then transferred to a solution of $0.1 \%$ Triton X-100 and 5\% normal goat serum in PBS for $30 \mathrm{~min}$. Sections were then incubated in a rabbit GFAP antibody (1:1000; Sapphire Bioscience) with $0.1 \%$ Triton X-100 and 5\% normal goat serum in PBS for $48 \mathrm{~h}$. Three $10 \mathrm{~min}$ PBS washes were given before sections were placed in a goat anti-rabbit Alexa 488 secondary antibody (1:200; Invitrogen) for $2 \mathrm{~h}$. Three $10 \mathrm{~min}$ PBS washes were then given before sections were mounted on slides with Fluromount.

Myelin was stained in a third series of sections containing amygdala structures to assess the damage to fibers in the target region. Sections were placed in FluoroMyelin Fluorescent Myelin Stain (1:300; Invitrogen) in PBS overnight, given three 10 min PBS washes and mounted with Fluromount. Determination of the extent of each lesion was made by a trained observer, who was blind to the subjects' group designation, using the boundaries defined by Paxinos and Watson (1998).

\section{Behavioral procedures}

As mentioned above, the behavioral procedures used in Experiments 1 and 2 had in common three distinct stages: lever press training, outcome devaluation, and an extinction test.

Lever-press training. Following 1 week of food deprivation, animals were given two sessions of magazine training. Sucrose solution was delivered at random $60 \mathrm{~s}$ intervals for 30 outcomes per session. Animals then received $8 \mathrm{~d}$ of instrumental training (two sessions per day) to press a single lever for sucrose solution delivery. Rats received three sessions of continuous reinforcement, four sessions of random interval of $15 \mathrm{~s}$ (RI15), four sessions of RI-30, and four sessions of RI-60. Right and left lever 
was counterbalanced across animals. Each session commenced with the insertion of the lever; sessions ended when 30 reinforcements were earned or after $60 \mathrm{~min}$, which ever came first.

Outcome devaluation. The day after the last session of training, the sucrose solution was devalued using conditioned taste aversion. All rats were given ad libitum access to sucrose solution for $30 \mathrm{~min}$ each day for $3 \mathrm{~d}$. Rats were placed in clear plastic tubs with bottles filled with sucrose solution attached. On each day, half of each type of lesion group received an intraperitoneal injection of lithium chloride $(0.15 \mathrm{M} \mathrm{LiCl}, 20 \mathrm{ml} / \mathrm{kg})$ and was placed back in their home cages, while the other half received saline injections $(20 \mathrm{ml} / \mathrm{kg})$. The amount of sucrose solution consumed each day was measured.

Extinction test. The day following the last day of outcome devaluation, all rats received a 5 min extinction test. The test began with the insertion of the same lever used during training and ended with the retraction of the lever. Lever presses and magazine entries were recorded, and no sucrose reward was delivered.

Pavlovian-instrumental transfer. After the extinction test, three groups of rats from Experiment 1 ( $11 \mathrm{aCeN}, 18 \mathrm{pCeN}$, and 8 shams) were tested for a general transfer effect. This involved three stages, which occurred as follows.

Pavlovian training. Rats were given eight daily sessions of Pavlovian training. Each session consisted of eight presentations of a 2 min tone [conditioned stimulus (CS+)], during which grain pellets were delivered on a random time of $30 \mathrm{~s}$ schedule. Intertrial intervals ranged from 2 to 4 min, with an average of $90 \mathrm{~s}$. The number of magazine entries during the stimulus and intertrial intervals was recorded.

Instrumental training. Rats were next given daily sessions of lever training to gain access to a grain pellet for 1 week. Sessions began with the insertion of the lever opposite to that used during the previous training and ended when 30 outcomes had been delivered or after $1 \mathrm{~h}$, whichever came first. Pellets were delivered on a fixed interval of $30 \mathrm{~s}$ schedule. Lever presses and magazine entries were recorded in each session.

Pavlovian reminder session. One session of Pavlovian training was given at the end of instrumental training, during which the CS + was presented six times, each presentation $2 \mathrm{~min}$ in duration. Additionally, two presentations of a 2 min white noise (CS-) were delivered randomly throughout the session. During these CS - presentations, no food pellets were delivered.

Transfer test. The following day, rats were tested for a general transfer effect in an extinction test. The test session began with the insertion of the lever used during instrumental training for the pellet outcome and remained out for the duration of the $48 \mathrm{~min}$ test. During the first $8 \mathrm{~min}$ of the session, no stimuli were presented. After this, four 2 min tones and four $2 \mathrm{~min}$ noise stimuli were randomly presented, with $3 \mathrm{~min}$ intertrial intervals. The number of lever presses was recorded during each stimulus presentation as well as during the 2 min preceding the stimuli, which served as the measure of baseline during the intertrial interval (ITI).

\section{Results}

We first compared responding during the test phase of the two parts of Experiment 1. ANOVA revealed no effect of Experiment in instrumental responding during the extinction test and no interactions involving this factor (all $F s<1$ ). As a consequence, we collapsed across these experiments for presentation and analysis.

\section{Histology}

Figure 1 provides schematic illustrations of the damage observed systematically in all animals in anterior $\mathrm{CeN}$ and (Fig. $1 \mathrm{~A}$ ) posterior CeN caused by ibotenic acid infusions (Fig. $1 B$ ), and DLS lesions caused by NMDA infusions (Fig. 1C). Photomicrographs of the lesions using fluorescent Nissl stain are provided in Figure 2 , middle and right columns, as well as their corresponding stereotaxic locations (Fig. 2, left). Generally, lesions to the posterior and anterior regions of the $\mathrm{CeN}$ resulted in extensive cell loss and shrinkage, as observed by the Nissl stain. DLS lesions resulted in extensive cell loss in this area, extending to the posterior DLS, but sparing the DMS and the globus pallidus. Lesions of the CeN also resulted in extensive astrocytic proliferation. Photomicrographs of anterior and posterior CeN sections stained with GFAP are provided in Figure 3, $A$ and B. Anterior (Fig. 3, left) and posterior (Fig. 3, right) $\mathrm{CeN}$ sections are provided for lesions aimed at anterior (Fig. $3 A$ ) and posterior (Fig. $3 B$ ) CeN. These figures suggests there was a small region of overlap between the posterior and anterior lesions of $\mathrm{CeN}$ as well as some dorsal damage along the needle track in each type of lesion. However, neither the region of overlap nor any apparent dorsal damage was systematically observed either bilaterally within subjects or between subjects in the lesioned groups. Importantly, there was little if any damage detected to the anterior $\mathrm{CeN}$ in posterior lesioned animals. Additionally, Figure $3 C$ provides representative sections from sham and lesioned animals stained with FluoroMyelin. It was clear that neither the microinjection procedure nor the neurotoxic lesions of the $\mathrm{CeN}$ caused by ibotenic acid resulted in damage to fibers passing through the $\mathrm{CeN}$, as can be seen by comparing sections from Sham (Fig. 3, left) and lesioned (Fig. 3, right) animals. In Experiment 1, five posterior and three anterior lesioned rats were excluded due to damage beyond the boundaries of these regions, resulting in the following group sizes: posterior, $n=41$; anterior, $n=19$; sham, $n=45$. Six animals in Experiment 2 were excluded, resulting in the following group sizes: contralateral, $n=23$; ipsilateral, $n=23$. Excluded animals were not included in the behavioral analysis.

\section{Experiment 1: Effect of anterior versus posterior $\mathrm{CeN}$ lesions on habit learning \\ Training}

All of the rats in the three lesioned groups acquired lever pressing and the groups increased performance over days. During the overtraining on the RI-60 schedule, the groups responded similarly, although the rats in Group aCeN appeared to respond at a slightly higher rate than the other groups. Mean responses per minutes during these last four sessions were as follows: $\mathrm{aCeN}$ devaluation (dev), 17.0; aCeN-non-devalued (non), 16.1; pCeNdev, 11.26; pCeN-non, 12.99; Sham-dev, 12.83; Sham-non, 12.58. Statistical analysis showed a main effect of lesion $\left(F_{(3,396)}=\right.$ $6.34, p<0.05$ ), indicating the anterior $\mathrm{CeN}$ groups responded at a higher rate, but there was no effect of devaluation and no groupby-devaluation interaction in these data (all $F_{S}<1$ ).

\section{Outcome devaluation: sucrose consumption}

The amount of sucrose solution consumed during the $3 \mathrm{~d}$ of taste aversion is shown in Figure 4A. Three-way ANOVA revealed that this amount significantly differed across devaluation (i.e., devalued vs non devalued; $\left.F_{(1,324)}=154.17, p<0.05\right)$ and days $\left(F_{(2,324)}=32.37, p<0.05\right)$. Further, there was a significant devaluation by day interaction $\left(F_{(2,324)}=39.07, p<0.05\right)$. That is, the devalued groups reduced their sucrose consumption across days whereas the non-devalued groups maintained a substantial level of consumption. The statistical analysis did not reveal any significant differences among the three devalued lesioned groups (all $F s<1$ ). There was, however, a lesion-by-devaluation interaction $\left(F_{(2,324)}=7.89, p<0.05\right)$. However, Fisher least square difference post hoc analysis revealed that the interaction was due to the fact that non-devalued rats with anterior CeN lesions drank slightly less sucrose than the two other non-devalued groups $(p<0.05)$. By the third session of devaluation, there was a significant devaluation effect in each of the lesioned groups when the devalued and non-devalued groups were compared: Groups 

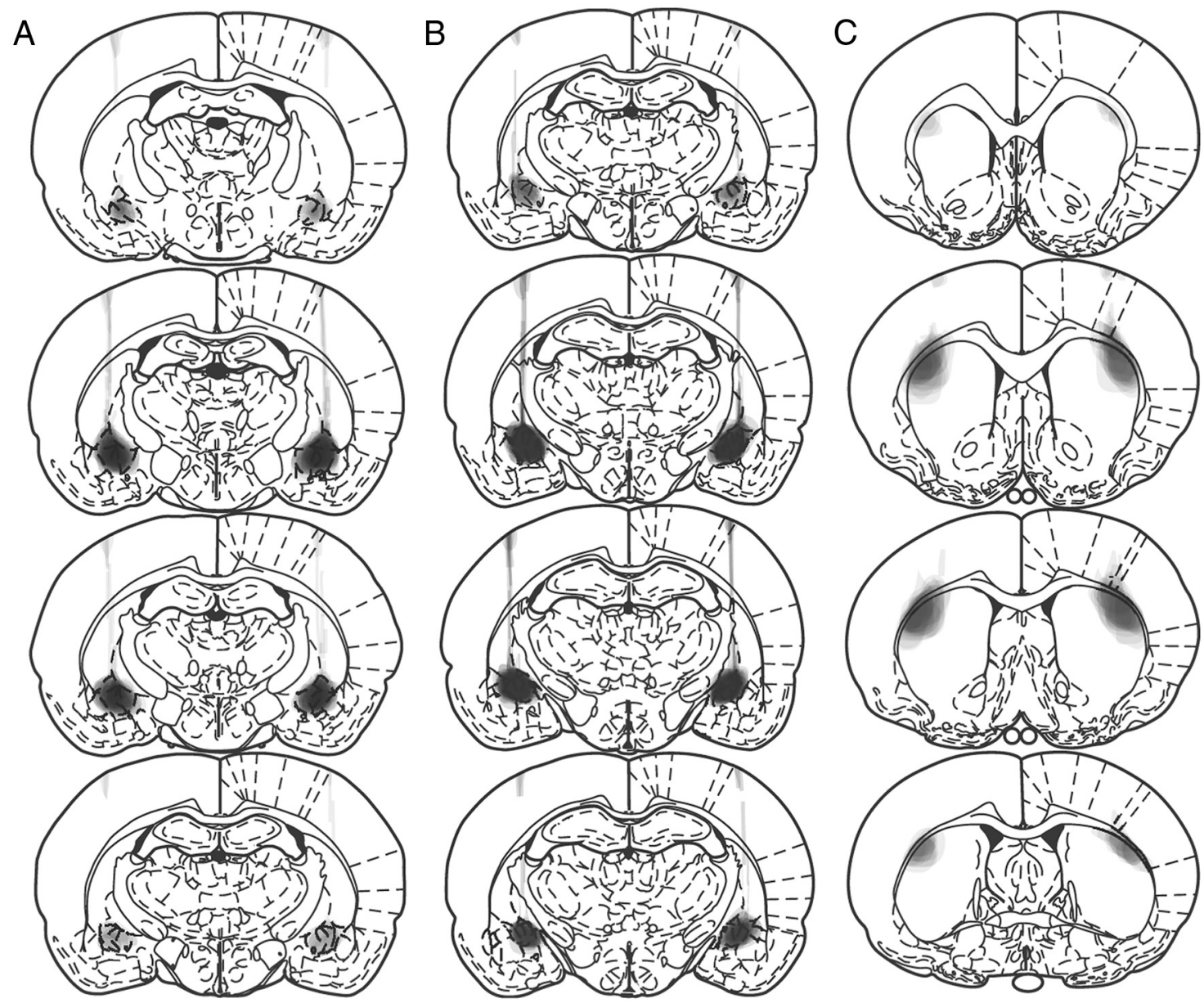

Figure 1. Schematic depiction of lesions to the anterior CeN (A), posterior CeN (B), and DLS (C). Stereotaxic locations are $-1.6,-1.8,-1.88$, and -2.12 for anterior CeN lesions; $-2.12,-2.3$, -2.56 , and -2.8 for posterior CeN lesions; $+1.6,+1.0,+0.48$, and -0.26 for DLS lesions. Shading indicates the extent of neuronal loss across subjects, with each subject represented as a separate, stacked layer. All coordinates are relative to bregma; sections are based on Paxinos and Watson (1995).

aCeN: $F_{(1,17)}=50.38, p<0.05 ;$ Groups pCeN: $F_{(1,39)}=143.19$, $p<0.05$; Groups Sham: $F_{(1,43)}=121.36, p<0.05$.

Outcome devaluation: extinction test

The effect of outcome devaluation on lever press performance was assessed as a change in performance from the overtraining baseline; i.e., the average rate of lever pressing during the last four sessions of training on the RI-60 schedule described above (Fig. $4 B)$. No obvious devaluation effect emerged in either of the control groups: pCeN-dev and pCeN-non responded similarly in the test, and if anything, Sham-dev responded slightly more than Sham-non. In contrast, rats given anterior CeN lesions appeared to show a devaluation effect with rats in Group aCeN-dev responding considerably less than those in aCeN-non. The statistical analysis was designed to test two contrasts: the effect of devaluation in the two control groups, Group Sham and Group $\mathrm{pCeN}$; and the effect of the anterior CeN lesions against performance in the two control groups. Two-way ANOVA conducted on the extinction test data in the control groups revealed no effect of lesion or of devaluation, and no lesion-by-devaluation interaction, confirming that the Sham and pCeN groups were insensitive to outcome devaluation (all $F s<1$ ). In contrast, ANOVA conducted to compare the control groups with the experimental group, Group aCeN, found a main effect of lesion $\left(F_{(1,101)}=5.99\right.$, $p<0.05)$ and, importantly, a significant lesion-by-devaluation interaction $\left(F_{(1,101)}=3.99, p<0.05\right)$. Simple effects analysis conducted on the significant interaction revealed a significant devaluation effect in Group aCeN $\left(F_{(1,18)}=7.43, p<0.05\right)$ but no effect of devaluation in either Group Sham $\left(F_{(1,44)}=1.99, p>\right.$ $0.05)$ or Group pCeN $\left(F_{(1,40)}=0.03, p>0.05\right)$. Lesions of the anterior $\mathrm{CeN}$ appeared, therefore, to attenuate habit learning and to produce instrumental performance that remained sensitive to changes in outcome value after overtraining.

\section{Transfer test}

To establish whether the lesions in posterior $\mathrm{CeN}$ were effective, we assessed the sensitivity of lever press performance to the excitatory effects of presentation of a CS + in a general Pavlovianinstrumental transfer test. Previous studies suggest that rats with lesions of the posterior CeN can often show deficits in tests of this kind (Hall et al., 2001; Holland and Gallagher, 2003). For this assessment, we collapsed the groups across the devaluation manipulation to examine the effects of posterior CeN lesions $(n=$ $18)$, anterior CeN lesions $(n=11)$, and sham lesions $(n=8)$ on this transfer effect.

Pavlovian training led to robust conditioned responding to the CS + in each of the three groups. Mean magazine entries during these stimuli during the final session of training were as follows: pCeN ITI, 6.36; pCeN CS+, 14.34; aCeN ITI, 4.80; aCeN CS+, 16.14; Sham ITI, 8.12; Sham CS +, 16.80. Responding did not differ in any of the groups. ANOVA revealed significantly higher levels of magazine entries during the CS+ presentation 

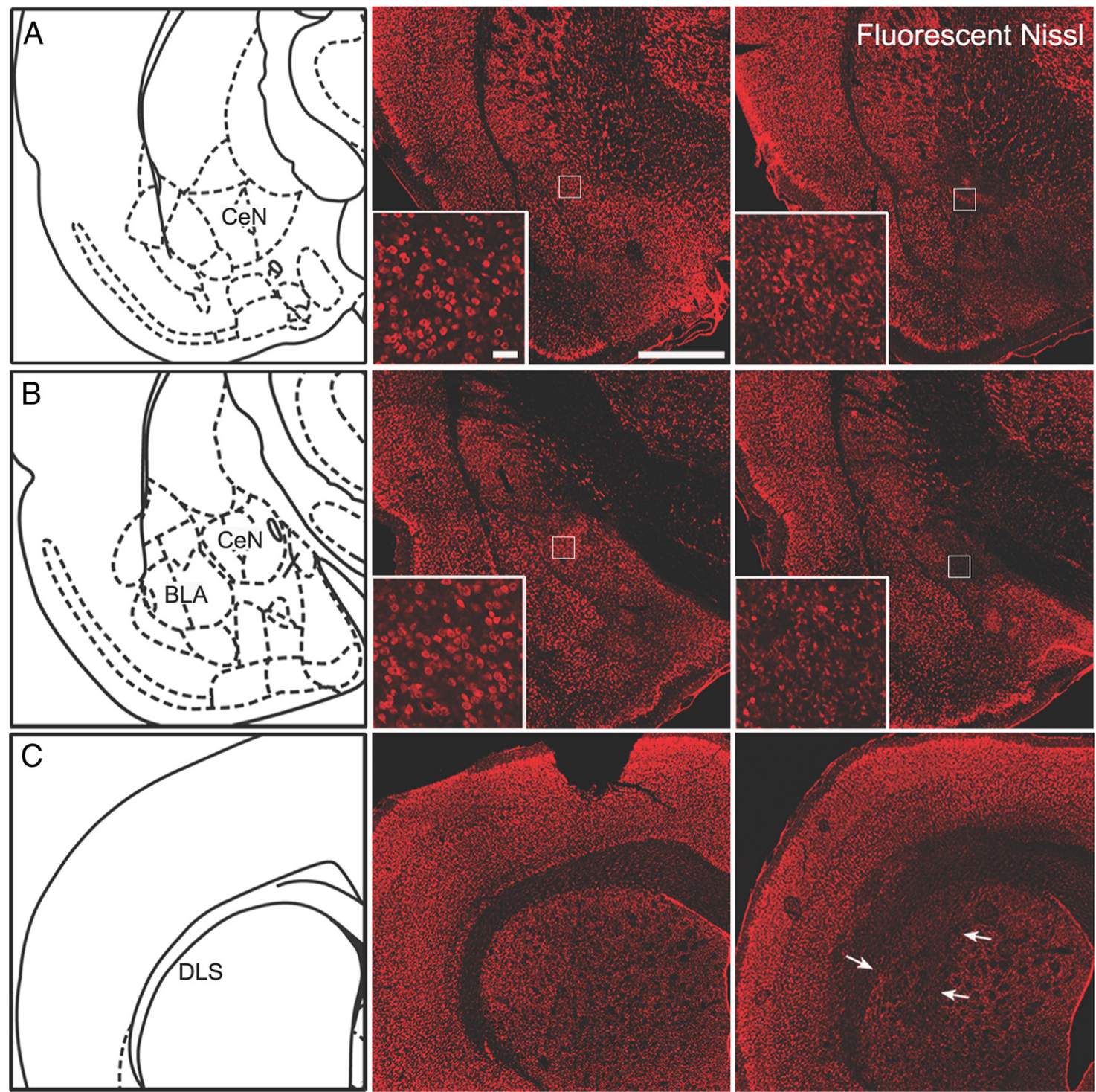

Figure 2. $A-C$, Left, Stereotaxic locations at $-1.8(\boldsymbol{A}),-2.3(\boldsymbol{B})$, and $+0.7 \mathrm{~mm}(\boldsymbol{C})$ relative to bregma, corresponding to anterior CeN, posterior CeN, and DLS, respectively. Middle and right, Photomicrographs of sham (middle) anterior CeN (A), posterior CeN (B), and DLS $(\boldsymbol{C})$ and their corresponding lesions (right). All sections were stained with fluorescent Nissl. Scale bar, $1 \mathrm{~mm}$; inset, $50 \mu \mathrm{m}$.

than during the ITI by the end of training $\left(F_{(1,68)}=43.64, p<\right.$ $0.05)$ but no effect of lesion $\left(F_{(2,68)}=0.08, p>0.05\right)$ and no interaction between lesion and CS type $\left(F_{(2,68)}=0.60, p>0.05\right)$.

The data from the Pavlovian-instrumental transfer test are presented in Figure 5. A clear positive transfer effect emerged in the Sham group; the CS+ produced a robust elevation in performance over baseline performance during the ITI. In contrast, the CS + appeared to have no effect on performance in either the anterior $\mathrm{CeN}$ lesioned group or the posterior $\mathrm{CeN}$ lesioned group; performance during the CS+ was similar to baseline. ANOVA conducted to compare responding among the lesion groups found a main effect of lesion $\left(F_{(2,68)}=6.01, p<0.05\right)$, of CS $\left(F_{(2,68)}=3.20, p<0.05\right)$, and a lesion-by-CS interaction $\left(F_{(2,68)}=3.20, p<0.05\right)$. Simple effects analysis revealed that responding during the $\mathrm{CS}+$ did not differ from the ITI for either Group aCeN $\left(F_{(1,21)}=0.01, p>0.05\right)$ or $\operatorname{pCeN~}\left(F_{(1,35)}=0.001\right.$, $p>0.05)$ but that the $\mathrm{CS}+$ significantly elevated responding over the ITI in Group Sham $\left(F_{(1,15)}=6.70, p<0.05\right)$, representative of a general transfer effect.
Experiment 2: Effect of amygdala-striatal disconnection on habit learning

Baseline training

During instrumental training, all of the rats acquired lever pressing and there were no differences in performance between the groups. Average rates of lever pressing during the last four sessions of training on the RI-60 schedule for the four groups were as follows: Ipsi-dev, 15.74; Ipsi-non, 13.20; Contra-dev, 13.71; Contra-non, 15.66. Two-way ANOVA conducted on these training data revealed no effect of group $\left(F_{(1,42)}=2.12, p>0.05\right)$, no effect of devaluation $\left(F_{(1,42)}=0.02, p>0.05\right)$, and no interaction between these factors $\left(F_{(1,42)}=3.67, p>0.05\right)$.

\section{Outcome devaluation: sucrose consumption}

Consumption data from taste aversion-induced outcome devaluation are presented in Figure 6A. This figure shows the development over the course of pairings of a similar taste aversion in the Ipsi- and Contra-dev groups relative to the non-devalued controls. Three-way ANOVA showed significant main effects of 
devaluation (i.e., devalued vs non-devalued; $\left.F_{(1,126)}=159.69, p<0.05\right)$ and days $\left(F_{(2,126)}=18.38, p<0.05\right)$ and a significant devaluation-by-day interaction $\left(F_{(2,126)}=34.95, p<0.05\right)$, indicating devalued rats reduced their consumption over days while non-devalued rats continued to consume the sucrose solution. There was no effect of lesion $\left(F_{(1,126)}=\right.$ $0.41, p>0.05)$ and no interactions involving lesions (largest $F_{(1,126)}=3.16$, $p>0.05)$.

Outcome devaluation: extinction test

The number of lever presses per minute during the extinction test is shown in Figure $6 B$ as a percentage of baseline responding. Overtraining produced clear habit learning as demonstrated by insensitivity to devaluation in the Ipsi-dev group as performance in that group was similar to that of Group Ipsi-non. In contrast, contralateral lesions of the aCeN and DLS produced a clear deficit in habit learning; the Contra-dev group appeared to respond less on test than Group Contra-non. Two-way ANOVA revealed neither a main effect of lesion or of devaluation but, critically, a significant lesion-by-devaluation interaction $\left(F_{(1,42)}=8.31, p<0.05\right)$. Simple effect analysis revealed a significant difference between the devalued and nondevalued rats in the contralateral group $\left(F_{(1,22)}=10.82, p<0.05\right)$ but no difference in the ipsilateral group $\left(F_{(1,22)}=\right.$ 1.05, $p>0.05$ ).

\section{Discussion}

The current results give further insight into the neural mechanisms that mediate habitual instrumental actions. We found clear evidence to suggest that the anterior portion of the central nucleus of the amygdala is involved in the acquisition of habits and that this region of $\mathrm{CeN}$ interacts with the DLS during the shift from goal-directed to habitual action control. Thus, although overtraining rendered sham-lesioned rats and rats with lesions of posterior CeN insensitive to the effects of taste aversion-induced outcome devaluation, bilateral lesions of the anterior $\mathrm{CeN}$ reduced this insensitivity and increased the influence of devaluation on the rats' instrumental performance without affecting performance when the outcome was not devalued. Hence, despite overtraining, goal-directed action control was maintained in the anterior CeN-lesioned group. Furthermore, lesions to the DLS made contralateral to the anterior CeN lesion produced a similar disruption in habit formation. These rats showed reliable devaluation and decreased responding in the extinction test after outcome devaluation in similar fashion to the bilateral anterior $\mathrm{CeN}$ lesioned rats. In contrast, when the DLS lesion was made ipsilateral to the CeN lesion, rats behaved like sham controls
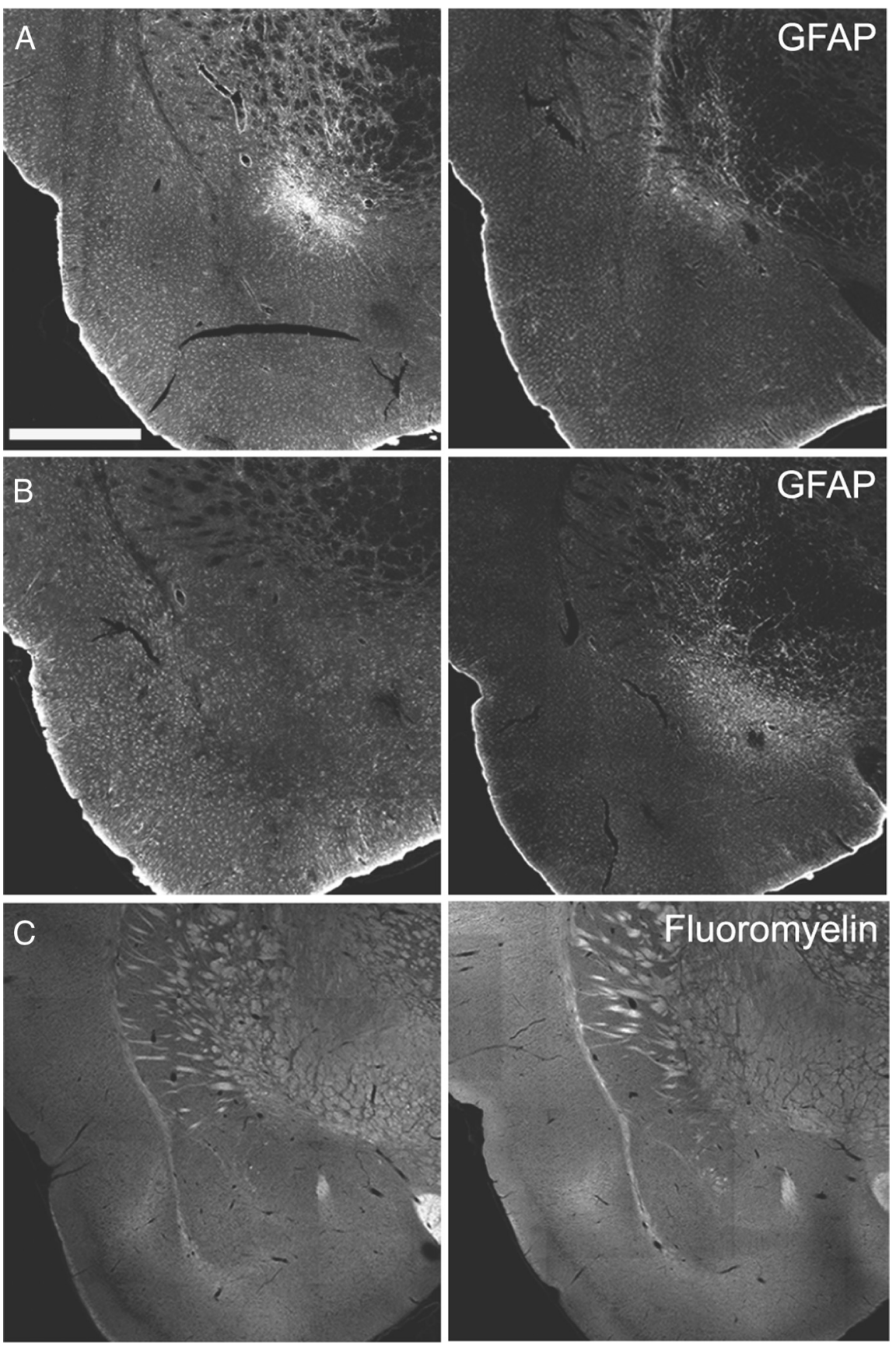

Figure 3. Anterior CeN lesion ( $-1.8 \mathrm{~mm}$ posterior to bregma; $\boldsymbol{A})$ and posterior CeN lesion $(-2.3 \mathrm{~mm}$ posterior to bregma; $\boldsymbol{B})$ stained with GFAP. Left, Representative anterior CeN sections $(-1.8 \mathrm{~mm}$ posterior to bregma). Right, Representative posterior CeN sections ( $-2.3 \mathrm{~mm}$ posterior to bregma). C, Anterior CeN ( $-1.8 \mathrm{~mm}$ posterior to bregma) sham (left) and lesion (right) sections stained with FluoroMyelin. Scale bar, $1 \mathrm{~mm}$.

continuing to press the lever despite devaluation; apparently, intact communication between the anterior CeN and the DLS in one hemisphere is adequate to induce sufficient habit learning to overcome the influence of devaluation.

It is important to note that the difference in the effects of the anterior and posterior CeN lesions on habit learning was not due to an ineffective posterior lesion; the general excitatory effects of a Pavlovian CS+ on instrumental performance were abolished in both the anterior and posterior $\mathrm{CeN}$-lesioned groups in the Pavlovian-instrumental transfer test. As Holland and Gallagher (2003) and Hall et al. (2001) have previously shown, lesions to the $\mathrm{CeN}$ (which correspond in our experiment to the posterior CeN) 
A

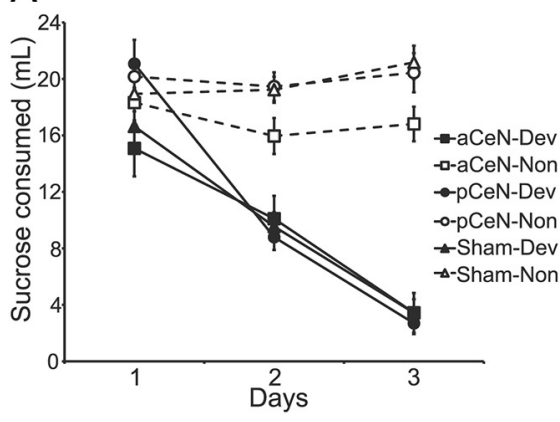

B

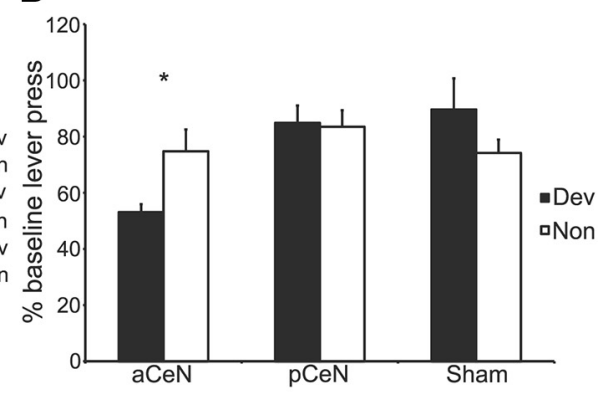

Figure 4. Bilateral anterior, but not posterior, CeN lesions disrupt the performance of normal habitual behavior after outcome devaluation. $\boldsymbol{A}$, The amount of sucrose consumed during the $3 \mathrm{~d}$ of conditioned taste aversion. $\boldsymbol{B}$, Results from the 5 min extinction test, plotted as a percentage of baseline responding during the last $4 \mathrm{~d}$ of training ( \pm 1 SEM). ${ }^{*} p<0.05$.

anterior CeN lesions had an effect on habit formation. Hence, in contrast to the conclusions derived from some behavioral studies (Holland, 2004), it appears that the Pavlovian incentive motivational process that mediates transfer is not necessary for habit formation, although habit formation may well be a necessary (though not sufficient) for transfer.

These experiments demonstrate the importance of both the central nucleus in the performance of habits and the interaction between the central nucleus and the dorsolateral striatum. Both the dorsolateral and dorsomedial parts of the striatum have been implicated in the performance of habitual and goal-directed actions;

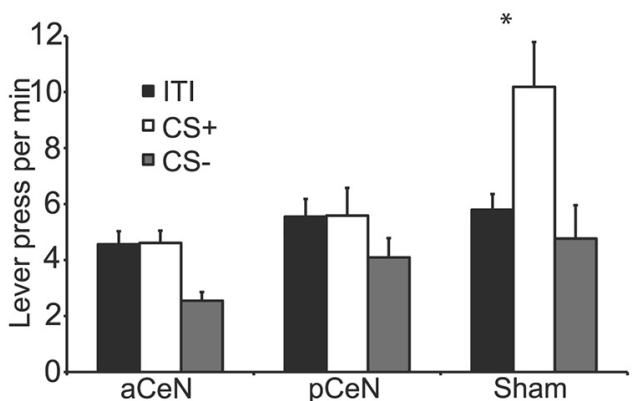

Figure 5. Effects of anterior and posterior lesions on Pavlovian-instrumental transfer. Sham rats exhibited normal Pavlovian-instrumental transfer. Responding increased during the CS that signaled the delivery of the outcome (CS+) compared with baseline (ITI). aCeN and pCeN showed no transfer; performance did not increase during the presentation of the $\mathrm{CS}+$ compared with ITI ( \pm 1 SEM). ${ }^{*} p<0.05$.
A

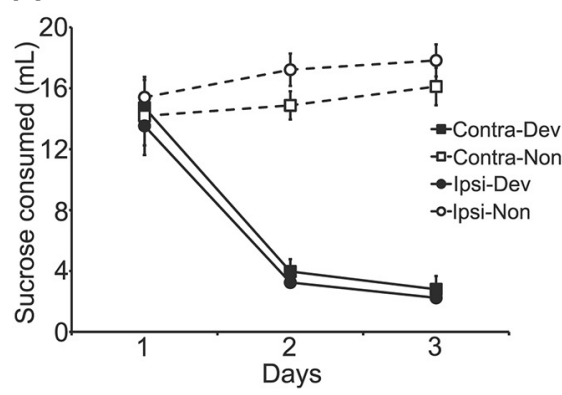

\section{B}

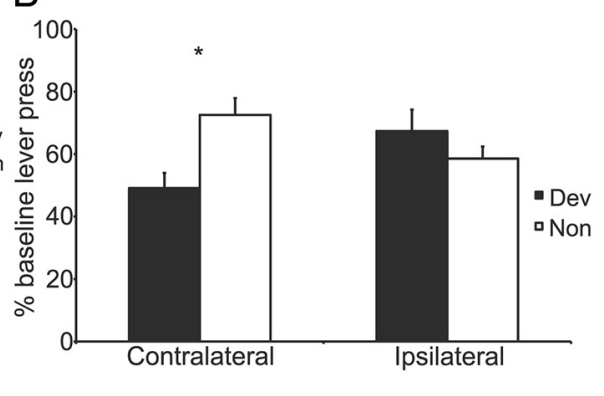

Figure 6. Disconnection of the anterior CeN from the DLS disrupts the performance of normal habitual behavior after outcome devaluation. $\boldsymbol{A}$, The amount of sucrose consumed during the $3 \mathrm{~d}$ of conditioned taste aversion. $\boldsymbol{B}$, Performance of rats with contralateral or ipsilateral lesions of the CeN and DLS during the 5 min extinction test, shown as a percentage of baseline responding during the last $4 \mathrm{~d}$ of training $\left( \pm 1\right.$ SEM). ${ }^{*} p<0.05$.

disrupt the ability of a Pavlovian CS to elevate instrumental performance. In our experiment, this effect was found in both groups given lesions of $\mathrm{CeN}$; i.e., although sham rats showed an increase in responding during the $\mathrm{CS}+$, neither the anterior nor the posterior CeN-lesioned rats showed this effect. Furthermore, the increase in responding in the Sham group was not due to the presentation of an extraneous stimulus but depended on the CS having been previously paired with an unconditioned stimulus; a neutral CS - failed to elevate performance over baseline, something also seen in the lesioned groups (Fig. 5). It is interesting to note, therefore, that, whereas both posterior and anterior CeN lesions had an effect on Pavlovian-instrumental transfer, only whereas the dorsomedial striatum guides actions based on the current value of their associated outcome, the dorsolateral striatum is important for the performance of habits (Yin et al., 2004, 2006). However, how the central nucleus and the dorsolateral striatum communicate during the course of overtraining is still unresolved. It is possible that the central nucleus maintains direct projections onto the striatum, as portions of the central nucleus, such as the striatal-amygdala transition, have a striatallike structure (McDonald, 1982; Cassell et al., 1986; Sah et al., 2003). However, although there is evidence that there are direct amygdala-striatal projections, these projections seem to extend mostly to the medial part and appear to spare the dorsolateral part of the striatum (Kelley et al., 1982).

Alternatively, it is possible that the central nucleus controls the DLS indirectly via some other structure. One possibility is the reasonably dense projection from the $\mathrm{CeN}$ to regions of the thalamus that then project topographically (and ipsilaterally) onto DLS (Sah et al., 2003). Perhaps a more plausible, certainly more attractive, suggestion is that the $\mathrm{CeN}$ controls the DLS through its influence over the ascending nigrostriatal dopamine projection. There is anatomical evidence that anterior portions of the $\mathrm{CeN}$ project to the substantia nigra pars compacta (Gonzales and Chesselet, 1990), and unpublished tracing studies from our lab confirm this. However, it is unclear whether the anterior $\mathrm{CeN}$ projection to the $\mathrm{SNc}$ is excitatory or inhibitory; stimulation of neurons in the $\mathrm{CeN}$ has been reported to directly modulate $\mathrm{SNc}$ responses, producing excitation in one population of dopamine neurons and inhibition in another (Rouillard and Freeman, 1995). It has been found that the majority of neurons in the CeN contain GAD 65 and 67 mRNA (Pitkänen and Amaral, 1994; Poulin et al., 2008), so it is likely that most outputs from the CeN are inhibitory. Indeed, in Rouillard and Freeman's (1995) study, the excitatory effects on SNc neurons of CeN stimulation have a much longer latency than the inhibitory effects. Furthermore, the neurons that were excited had a much lower basal firing rate, suggesting they were under tonic inhibition. Hence, CeN stimulation appears to have both a direct inhibitory effect on one population of dopamine neurons and an indirect excitatory effect on another population, likely through inhibition of local GABAergic interneurons (Chuhma et al., 2011). 
One of the more intriguing aspects of these results is the implication that they have for the role of the amygdala in instrumental conditioning. As mentioned in the Introduction above, the BLA, but not the CeN (Corbit and Balleine, 2005), has been consistently implicated in the incentive learning that mediates the reward process associated with goal-directed action (Balleine et al., 2003; Wang et al., 2005; Wassum et al., 2009, 2011). The current results provide evidence that, although the CeN may not be involved in this reward process, it plays a clear role in habit learning, potentially through control of the reinforcement signal provided to the dorsal striatum by the midbrain dopaminergic projection. To the extent this is true, a degree of symmetry may be established within the amygdala with the BLA mediating reward and the CeN [likely as a component of the extended amygdala (Fudge and Emiliano, 2003)] mediating reinforcement. It has long been recognized that the BLA and CeN receive distinct sources of information, the BLA more sensory specific and the $\mathrm{CeN}$ more emotionally general inputs, and have, therefore, been argued to serve distinct functions at least in instrumental conditioning if not in other forms of learning (Blundell et al., 2001; Balleine and Killcross, 2006). Despite this difference, however, it appears that the amygdala can be described as having a more general function in instrumental conditioning, serving to parse the instrumental outcome into two distinct feedback signals critical for the acquisition of goal-directed and habit-learning processes (for review, see Balleine and Killcross, 2006; Balleine et al., 2008; 2009; Balleine and O'Doherty, 2010). This analysis proposes, therefore, that distinct nuclei within the amygdala encode different aspects of the instrumental outcome and function to promote distinct learning processes through their influence on anatomically and functionally distinct circuits in dorsal striatum.

A second implication of the current results is also worth raising here. One of the outstanding issues in instrumental conditioning is the relationship between goal-directed and habitlearning processes. Generally, these have been argued to be mutually exclusive, yet it is important to note that they often seem to interact in a competitive manner; inactivation of either the DMS or of the BLA can immediately render instrumental responding habitual (Yin et al., 2005b; Ostlund and Balleine, 2008), suggesting that, early in training, habitual control is under some form of tonic inhibition. Although hypotheses concerning this interaction have largely focused on the basal ganglia (Balleine et al., 2008), there is a growing body of literature documenting the interaction between BLA and CeN and it is possible that important aspects of the inhibitory interaction between goaldirected and habitual control are mediated by this interaction. This suggestion is particularly appealing because current evidence suggests that any inhibitory relationship between BLA and regions of the $\mathrm{CeN}$ is likely regulated by the intercallated cell masses (Ehrlich et al., 2009; Busti et al., 2011) that lie in the margin between BLA and CeN, which themselves appear to be regulated by the infralimbic cortex (Li et al., 2011), an area that has been previously implicated in the interaction between goaldirected and habitual control of performance (Coutureau and Killcross, 2003).

Whatever the role of the interaction of the BLA and CeN turns out to be, the current results provide the first evidence that the amygdala, specifically the CeN, plays a role in the acquisition of habits. Furthermore, we have demonstrated that the role of the $\mathrm{CeN}$ depends on its interaction with the DLS, a region previously implicated in the acquisition and control of habitual actions (Yin et al., 2004; Graybiel, 2008).

\section{References}

Adams CD (1982) Variations in the sensitivity of instrumental responding to reinforcer devaluation. Q J Exp Psychol 34:77-98.

Adams CD, Dickinson A (1981) Instrumental conditioning following reinforcer devaluation. Q J Exp Psychol 33:109-121.

Balleine BW, Dickinson A (1998) Goal-directed instrumental action: contingency and incentive learning and their cortical substrates. Neuropharmacology 37:407-419.

Balleine BW, Killcross S (2006) Parallel incentive processing: an integrated view of amygdala function. Trends Neurosci 29:272-279.

Balleine BW, O'Doherty JP (2010) Human and rodent homologies in action control: corticostriatal determinants of goal-directed and habitual action. Neuropsychopharmacology 35:48-69.

Balleine BW, Killcross AS, Dickinson A (2003) The effect of lesions of the basolateral amygdala on instrumental conditioning. J Neurosci 23:666-675.

Balleine BW, Daw ND, O’Doherty J (2008) Multiple forms of value learning and the function of dopamine. In: Neuroeconomics: Decision making and the brain (Glimcher P, Camerer C, Fehr E, Poldrack R, eds), pp 367-387. London: Academic.

Balleine BW, Liljeholm M, Ostlund SB (2009) The integrative function of the basal ganglia in instrumental conditioning. Behav Brain Res 199:43-52.

Blundell P, Hall G, Killcross S (2001) Lesions of the basolateral amygdala disrupt selective aspects of reinforcer representation in rats. J Neurosci 21:9018-9026.

Busti D, Geracitano R, Whittle N, Dalezios Y, Mańko M, Kaufmann W, Sätzler K, Singewald N, Capogna M, Ferraguti F (2011) Different fear states engage distinct networks within the intercalated cell clusters of the amygdala. J Neurosci 31:5131-5144.

Cassell MD, Gray TS, Kiss JZ (1986) Neuronal architecture in the rat central nucleus of the amygdala: a cytological, hodological, and immunocytochemical study. J Comp Neurol 246:478-499.

Chuhma N, Tanaka KF, Hen R, Rayport S (2011) Functional connectome of the striatal medium spiny neuron. J Neurosci 31:1183-1192.

Corbit LH, Balleine BW (2005) Double dissociation of basolateral and central amygdala lesions on the general and outcome-specific forms of Pavlovian-instrumental transfer. J Neurosci 25:962-970.

Coutureau E, Killcross S (2003) Inactivation of the infralimbic prefrontal cortex reinstates goal-directed responding in overtrained rats. Behav Brain Res 146:167-174.

Dayan P, Balleine BW (2002) Reward, motivation, and reinforcement learning. Neuron 36:285-298.

Dickinson A, Balleine BW, Watt A, Gonzalez F, Boakes R (1995) Motivational control after extended instrumental training. Anim Learn Behav 23:197-206.

Ehrlich I, Humeau Y, Grenier F, Ciocchi S, Herry C, Lüthi A (2009) Amygdala inhibitory circuits and the control of fear memory. Neuron 62:757-771.

El-Amamy H, Holland PC (2006) Substantia nigra pars compacta is critical to both the acquisition and expression of learned orienting of rats. Eur J Neurosci 24:270-276.

Faure A, Haberland U, Condé F, El Massioui N (2005) Lesion to the nigrostriatal dopamine system disrupts stimulus-response habit formation. J Neurosci 25:2771-2780.

Fudge JL, Emiliano AB (2003) The extended amygdala and the dopamine system: another piece of the dopamine puzzle. J Neuropsychiatry Clin Neurosci 15:306-316.

Gonzales C, Chesselet MF (1990) Amygdalonigral pathway: an anterograde study in the rat with Phaseolus vulgaris leucoagglutinin (PHA-L). J Comp Neurol 297:182-200.

Graybiel AM (2008) Habits, rituals, and the evaluative brain. Ann Rev Neurosci 31:359-387.

Hall J, Parkinson JA, Connor TM, Dickinson A, Everitt BJ (2001) Involvement of the central nucleus of the amygdala and nucleus accumbens core in mediating Pavlovian influences on instrumental behaviour. Eur J Neurosci 13:1984-1992.

Holland PC (2004) Relations between Pavlovian-instrumental transfer and reinforcer devaluation. J Exp Psychol Anim Behav Process 30:104-117.

Holland PC, Gallagher M (2003) Double dissociation of the effects of lesions of basolateral and central amygdala on conditioned stimulus-potentiated 
feeding and Pavlovian-instrumental transfer. Eur J Neurosci 17: $1680-1694$.

Kelley AE, Domesick VB, Nauta WJ (1982) The amygdalostriatal projection in the rat: an anatomical study by anterograde and retrograde tracing methods. Neuroscience 7:615-630.

Li G, Amano T, Pare D, Nair SS (2011) Impact of infralimbic inputs on intercalated amygdala neurons: a biophysical modeling study. Learn Mem 18:226-240.

McDonald AJ (1982) Cytoarchitecture of the central amygdaloid nucleus of the rat. J Comp Neurol 208:401-418.

Ostlund SB, Balleine BW (2008) Differential involvement of the basolateral amygdala and mediodorsal thalamus in instrumental action selection. J Neurosci 28:4398-4405.

Paxinos G, Watson C (1998) The rat brain in stereotaxic coordinates, 4th edition. New York: Academic.

Pitkänen A, Amaral DG (1994) The distribution of GABAergic cells, fibers, and terminals in the monkey amygdaloid complex: an immunohistochemical and in situ hybridization study. J Neurosci 14:2200-2224.

Poulin JF, Castonguay-Lebel Z, Laforest S, Drolet G (2008) Enkephalin coexpression with classic neurotransmitters in the amygdaloid complex of the rat. J Comp Neurol 506:943-959.

Reynolds JN, Hyland BI, Wickens JR (2001) A cellular mechanism of reward-related learning. Nature 413:67-70.

Rouillard C, Freeman AS (1995) Effects of electrical stimulation of the central nucleus of the amygdala on the in vivo electrophysiological activity of rat nigral dopaminergic neurons. Synapse 21:348-356.
Sah P, Faber ES, Lopez De Armentia M, Power J (2003) The amygdaloid complex: anatomy and physiology. Physiol Rev 83:803-834.

Shiflett MW, Brown RA, Balleine BW (2010) Acquisition and performance of goal-directed instrumental actions depends on ERK signaling in distinct regions of dorsal striatum in rats. J Neurosci 30:2951-2959.

Wang SH, Ostlund SB, Nader K, Balleine BW (2005) Consolidation and reconsolidation of incentive learning in the amygdala. J Neurosci $25: 830-835$

Wassum KM, Ostlund SB, Maidment NT, Balleine BW (2009) Distinct opioid circuits determine the palatability and the desirability of rewarding events. Proc Natl Acad Sci U S A 106:12512-12517.

Wassum KM, Cely IC, Balleine BW, Maidment NT (2011) Mu-opioid receptor activation in the basolateral amygdala mediates the learning of increases but not decreases in the incentive value of a food reward. J Neurosci 31:1591-1599.

Yin HH, Knowlton BJ, Balleine BW (2004) Lesions of dorsolateral striatum preserve outcome expectancy but disrupt habit formation in instrumental learning. Eur J Neurosci 19:181-189.

Yin HH, Knowlton BJ, Balleine BW (2005a) Blockade of NMDA receptors in the dorsomedial striatum prevents action-outcome learning in instrumental conditioning. Eur J Neurosci 22:505-512.

Yin HH, Ostlund SB, Knowlton BJ, Balleine BW (2005b) The role of the dorsomedial striatum in instrumental conditioning. Eur J Neurosci 22:513-523.

Yin HH, Knowlton BJ, Balleine BW (2006) Inactivation of dorsolateral striatum enhances sensitivity to changes in the action-outcome contingency in instrumental conditioning. Behav Brain Res 166:189-196. 